\title{
Observation of Discrete, Vortex Light Bullets
}

\author{
Falk Eilenberger, ${ }^{1}$ Karin Prater, ${ }^{1}$ Stefano Minardi, ${ }^{1}$ Reinhard Geiss, ${ }^{1}$ Ulrich Röpke, ${ }^{2}$ Jens Kobelke, ${ }^{2}$ Kay Schuster, ${ }^{2}$ \\ Hartmut Bartelt, ${ }^{2}$ Stefan Nolte, ${ }^{1}$ Andreas Tünnermann, ${ }^{1}$ and Thomas Pertsch ${ }^{1}$ \\ ${ }^{1}$ Institute of Applied Physics, Abbe Center of Photonics, Friedrich-Schiller-Universität Jena, Max-Wien-Platz 1, 07743 Jena, Germany \\ ${ }^{2}$ Institute of Photonic Technology, Albert-Einstein-Strasse 9, 07745 Jena, Germany \\ (Received 28 August 2013; revised manuscript received 5 November 2013; published 18 December 2013)
}

\begin{abstract}
We report the first experimental observation of vortex light bullets that are discrete, spatiotemporal, solitary waves with orbital angular momentum. We analyze conditions for their existence and investigate their rich properties and dynamics. Vortex light bullets are excited in fiber arrays with spatially shaped femtosecond pulses and analyzed with a spatiotemporal cross correlator. Most importantly, we find that they have entirely new stability properties, being robust against considerable degrees of perturbation in a limited range of energies. All experimental findings are backed up by rigorous simulations, giving further insight into the rich dynamics of vortex light bullets.
\end{abstract}

DOI: 10.1103/PhysRevX.3.041031

\section{INTRODUCTION}

Solitary waves $[1,2]$ are among the most fascinating nonlinear wave phenomena. They constitute wave packets that are immune to linear broadening induced by dispersion and/or diffraction and are of strictly finite energy. They can be found in such diverse systems as ultracold atoms [3], ocean waves [4], the nervous system [5], or atmospheric phenomena [6]. Nonlinear optics, however, has traditionally pioneered experimental solitary-wave research $[7,8]$ due to a combination of flexible, high-quality wave-propagation environments and ready accessibility of experimental data, exploiting advanced excitation and analysis techniques.

The quest for the understanding of solitary-wave dynamics has led to the generation of solitary waves in ever more advanced environments and of increasing complexity. Of particular interest are solitary waves in media with a periodically modulated refractive index [9-11], called discrete solitons. The prediction [12] and observation [13] of discrete solitons $[10,11,14]$ in one-dimensional lattices soon led to the demonstration of higher-dimensional discrete solitons. Among these works have been the prediction [15-17] and observation of spatial solitons [18-20] and spatial vortex solitons $[21,22]$ in two-dimensional waveguide arrays and spatiotemporal quasisoliton pulses traveling in waveguiding structures with one transverse spatial dimension [23,24]. In particular, vortex solitons [25] have recently gained a considerable amount of interest because optical-angular-momentum fields are currently discussed as possible carriers of data and energy due to their favorable diffraction properties and low cross talk [26,27].

Published by the American Physical Society under the terms of the Creative Commons Attribution 3.0 License. Further distribution of this work must maintain attribution to the author(s) and the published article's title, journal citation, and DOI.
Subject Areas: Nonlinear Dynamics, Optics, Photonics

The arguably most complex solitary wave observed so far was the so-called light bullet [28] (LB), a spatiotemporal, solitary wave in a two-dimensional waveguide array [29]. As opposed to previous works, it is fully self-confined along all spatial and temporal transverse dimensions. It was predicted in the 1990s [30] but eluded observation [31] until recent breakthroughs in modeling [32,33], sample fabrication [34,35], and analysis techniques [36,37] could be brought to bear, immediately bringing forward new spatiotemporal effects such as self-induced soliton decay, direct space-time coupling, and superluminal decay [38,39].

Here, we present the first experimental observation of an even more complex, spatiotemporal, solitary wave: the discrete vortex light bullet (VLB), an angular-momentumstabilized compound of three fundamental LBs. We show that the VLB exhibits a new mode of partial stability, characterized by spatiotemporal desynchronization into its fundamental constituents upon long-range propagation. Then, we present evidence of its experimental observation and use experimental and numerical data to explore its internal dynamics, which is characterized by an intricate balance of angular momentum, discreteness, nonlinearity, and the dispersive properties of the medium.

\section{VORTEX LIGHT BULLETS AND SELF-DESYNCHRONIZATION}

To get a first insight into the properties of VLBs, we find VLB solutions of the idealized discrete-continuous, nonlinear Schrödinger equation with Kerr nonlinearity [38]. The term discreteness refers to the spatial dependence of the field, which is described as a superposition of fundamental modes with amplitudes $a_{n m}$ at the discrete sites of waveguides with index $(m, n)$ in a two-dimensional, hexagonal array in a tight-binding approximation. The tunneling of light from one waveguide mode to its neighbors' modes gives rise to a propagation behavior similar to diffraction that is hence termed "discrete diffraction." 
Of particular interest to this work is the robustness [30] of discrete diffraction against self-induced wave collapse [40], which hinders observation of LBs in homogenous materials [28]. This robustness is related to the different shape of the band structure for large transverse wave numbers, as compared to the homogeneous case. The waveguide array is experimentally realized as a fiber array [35], discussed in more detail in Appendix C and depicted in the inset of Fig. 1. The equation reads

$$
\begin{aligned}
i \frac{\partial a_{n m}(t, z)}{\partial z}= & \beta_{2} \frac{\partial^{2} a_{n m}(t, z)}{\partial t^{2}}+\gamma\left|a_{n m}(t, z)\right|^{2} a_{n m}(t, z) \\
& +c\left[a_{n+1 m}(t, z)+a_{n m+1}(t, z)\right. \\
& +a_{n+1 m+1}(t, z)+a_{n-1 m}(t, z) \\
& \left.+a_{n m-1}(t, z)+a_{n-1 m-1}(t, z)\right]
\end{aligned}
$$

where $a_{n m}$ is the amplitude in the $n m$ th core of the fiber array in the comoving frame of reference. $z$ is the propagation length, and $t$ is time. The parameters $\beta_{2}=$ $27 \times 10^{3} \mathrm{fs}^{2} / \mathrm{m}, c=28 \mathrm{~m}^{-1}$, and $\gamma=0.28 \times 10^{-3}(\mathrm{~mW})^{-1}$ are determined by the array geometry (see Appendix A) and, respectively, mediate the strength of dispersion, nearest-neighbor coupling, and nonlinearity. Cores at the edge of the 91-core array have fewer coupling partners according to their position. Parameter scaling of the

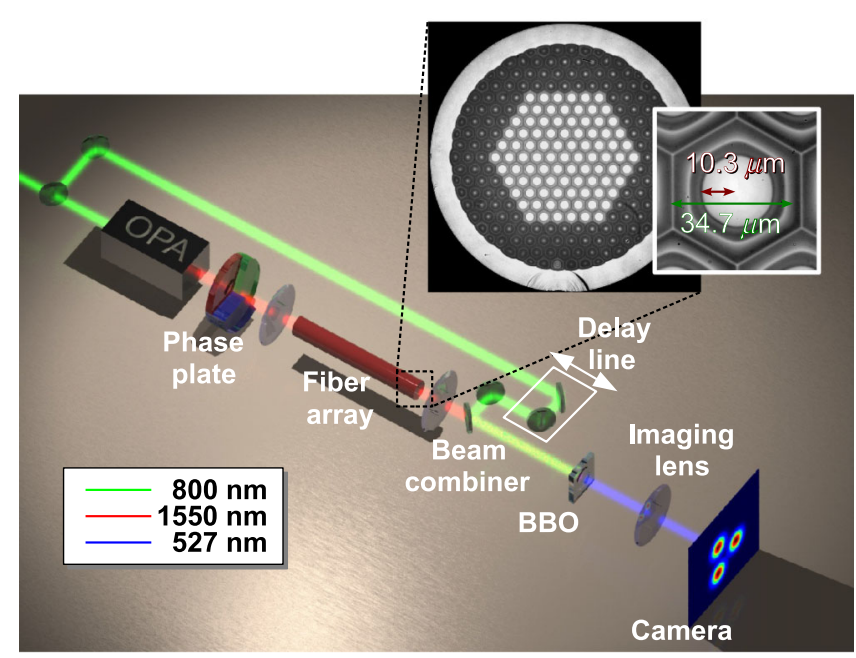

FIG. 1. Scheme of the experiment. Beam propagation is left to right. An 800-nm pulse (green line) is split. The first part bypasses the experiment through a delay line and impinges on a BBO crystal. The second is converted to $1550 \mathrm{~nm}$ in an optical parametrical amplifier (red line). It propagates through a phase plate, which imprints the discrete angular momentum on the pulse before it is focused onto the front of the fiber array, exciting a VLB. A second lens images the output onto the BBO crystal. This crystal generates sum-frequency light (blue line), which is imaged onto a $\mathrm{CCD}$, recording cross-correlation data proportional to the intensity $I(x, y, t)$ at the fiber array end. Inset: Microscope image of the fiber array front with (second inset) geometrical parameters of the unit cell. solutions is discussed in Ref. [38]. Note that Eq. (1) is a simplified, prototypical model, serving as a minimal system to study the interplay of dispersion, diffraction, nonlinearity, and optical angular momentum. It does not include the higher-order effects discussed below, which are needed to understand the full dynamics of VLBs, but it is a helpful tool to understand the basic mechanisms of VLB propagation and stability.

Nontrivial stationary solutions with $a_{n m}(z, t)=\tilde{a}_{n m}(t) \times$ $\exp (i b z)$ to Eq. (1) are found with a Newton-Raphson scheme. The specific type of LB is selected by picking initial conditions with the desired symmetry. The physical meaning of the family parameter $b$ is that of a nonlinearly induced offset of the longitudinal wave number $\beta$. It is therefore commonly referred to as the nonlinear phase shift $b$. We solve for VLBs, for single LBs, concentrated in a single waveguide, and for triplet LBs, which have the same field in three neighboring waveguides but no angular momentum. While Fig. 2 shows that all three solutions have qualitatively similar properties, we find that both single as well as triplet LBs have the same cutoff phase shift $b_{\text {cut }}=175 \mathrm{~m}^{-1}$; the VLB, however, has a significantly reduced $b_{\text {cut }}=160 \mathrm{~m}^{-1}$. All three solutions have a threshold energy $E_{\text {thresh }}$ at a particular $b_{\text {thresh }}$, suggesting that they are all subject to redshift-induced decay, first described for ordinary LBs [31]. For any nonlinear phase shift $b$, we find that the energy of the VLB is higher than 3 times the energy of the single LB.

Stability analysis is carried out by propagating the stationary solutions with numerical noise using Eq. (1). Noise sources are uncertainties of the initial conditions and errors induced by the numerical integrator. Their impact has been tested by the variation of the temporal resolution, the integrator step size, and the addition of white noise to the initial conditions. The results do not depend, within certain

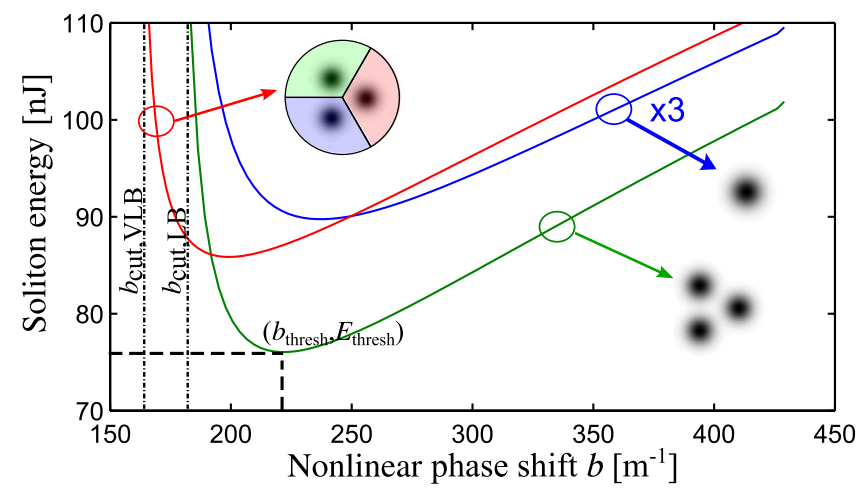

FIG. 2. Families of various LB solutions of the nonlinear Schrödinger equation [Eq. (1)]. Displayed is the energy $E$ vs the nonlinear phase shift $b$. The red line denotes the VLB. The green line denotes a triplet LB. The blue line denotes a single LB (energy multiplied by 3 ). The dashed black line denotes the minimal energy $E_{\min }$ and the corresponding nonlinear phase shift $b_{\text {min }}$. The dash-dotted black line denotes the solution with the minimal nonlinear phase shift $b_{\text {cut }}$. 
limits, on the strength of either of these noise sources. It is found for single LBs [41] that those with $b<b_{\text {thresh }}$ are unstable whereas all others are stable. Triplet LBs are never stable and are subject to modulation instability.

For VLBs, however, we find semistable solutions for $b>b_{\text {crit }}=557 \mathrm{~m}^{-1}$ and unstable solutions otherwise [see Fig. 3(a)]. While the unstable solutions exhibit exponential perturbation growth, as expected from modulation instability, semistable VLBs retain their shape for a considerable propagation length $z \approx 140 \mathrm{~mm}$ and then decay suddenly [see Fig. 3(b)]. Apart from nonexponential noise growth behavior, we also observe different decay products. Unstable solutions decay into a single LB and dispersive waves [Fig. 3(c)]. The three main peaks of semistable VLBs, however, desynchronize into three temporally separated LBs without dispersive background [Fig. 3(d)]. This desynchronization decay is another novel, nonlinearitytriggered, spatiotemporal transition [39] of LBs. It coincides with the findings of Ref. [42], predicting similar results for square arrays. Our findings complement those in Ref. [43], predicting a slightly lower value for $b_{\text {crit }} \approx$ $476 \mathrm{~m}^{-1}$ and stability for all $b>b_{\text {crit }}$; the difference might be due to the "temporal filtering" method employed by the authors in Ref. [42].

We mention that for all experimental purposes, semistable VLBs can be considered stable, as desynchronization would happen on lengths of $z \approx 100-200 \mathrm{~mm}$, equating into more than 30 dispersion lengths $L_{D}$. Decay by means of self-induced redshift does occur much earlier [31,38], as discussed below.
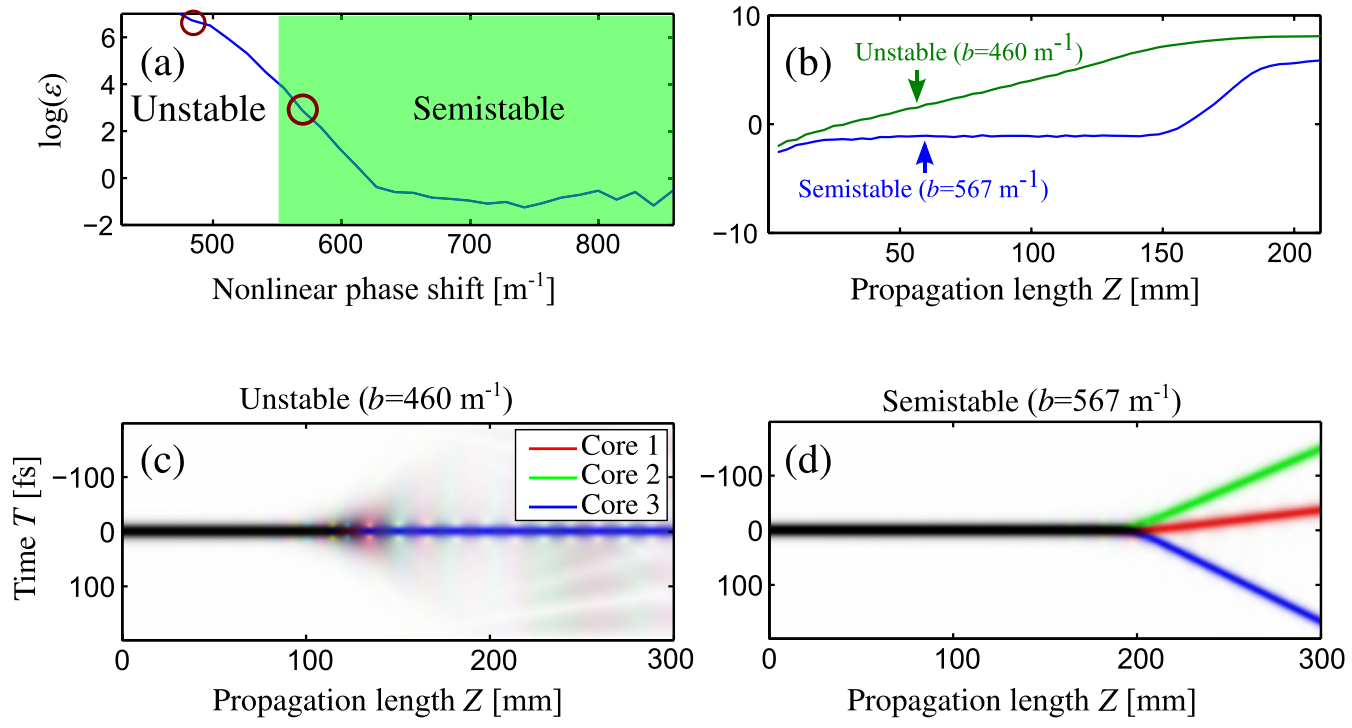

FIG. 3. Stability of the VLB solutions of Eq. (1). (a) Perturbation $\log (\varepsilon)$ at $Z=100 \mathrm{~mm}$, with a stability transition occurring at $b \approx 550 \mathrm{~m}^{-1}$. More details are given in (b)-(d) for the circled values. The definition of $\log (\varepsilon)$ is given in Appendix D. (b) Growth of perturbation $\log (\varepsilon)$ as function of propagation length. Exponential growth for the unstable solution is represented by the green region, and sudden onset growth for the semistable solution is represented by the solid blue line. (c),(d) Evolution of the temporal pulse intensity for the three main cores for (c) an unstable solution and (d) a semistable solution. The cores are color coded into the red-, green-, and blue-color (RGB) channels, respectively. 


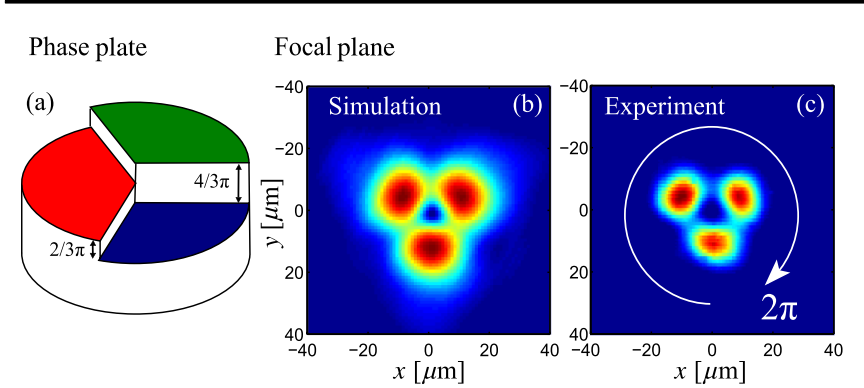

Discrete diffraction
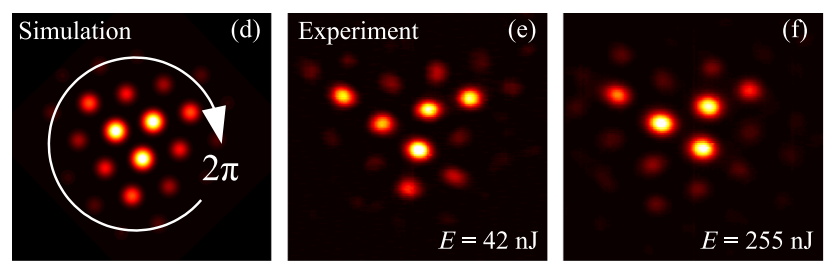

FIG. 4. (a) Phase-plate design. (b) Simulated and (c) experimental images of the focal spot intensity produced by a Gaussian beam and the phase plate. Adjacent peaks have $2 / 3 \pi$ phase difference. (d)-(f) Diffraction patterns of discrete vortices in a hexagonal lattice of $17-\mathrm{mm}$ length $\left(c=28 \mathrm{~m}^{-1}\right)$. (d) Simulated and (e) experimental discrete diffraction patterns in the linear, low power range. (e) Experimental pattern in the VLB power range. Increased contrast of the center compared to the outer waveguides is observed.

observed in the energy range of $250 \mathrm{~nJ}<E<300 \mathrm{~nJ}$, giving a first hint on the occurrence of VLBs. This range is consistent with later results.

It is now instructive to validate the findings of Sec. II with a realistic numerical model, including higher-order effects, such as higher-order dispersion, chromatic variation of the discrete coupling, and higher-order nonlinearity. The model is based on the solution of the unidirectional Maxwell equations [46,47] and discussed in Ref. [38]. In particular, we pay attention to the semistability of the VLBs and their robustness against residual excitation asymmetry, which is evident from Figs. 4(d) and 4(e).

To differentiate between the two mechanisms that might perturb the VLBs, we run two sets of simulations. First, we inject three $2 \pi / 3$ phase-shifted, otherwise identical Gaussian pulses with a FWHM of 50 fs into the array and determine the threshold energy needed to excite a VLB. This energy cannot be taken directly from the above discussed ideal solutions, as the excitation pulse length is considerably larger than the VLB duration and pulse shortening is accompanied by dispersive-wave generation [31].

At a threshold of $E_{\min }=260 \mathrm{~nJ}$, we find an increase of the light localization by roughly $20 \%$. An overview over the typical propagation dynamics in this energy range is given in Fig. 5. We observe temporally synchronized, subdispersive propagation [see Fig. 5(c)] of a pulse with duration of less than $25 \mathrm{fs}$ [Fig. 5(b)], if the sample length is shorter than $Z_{\max }<17 \mathrm{~mm}$. The pulses in all three
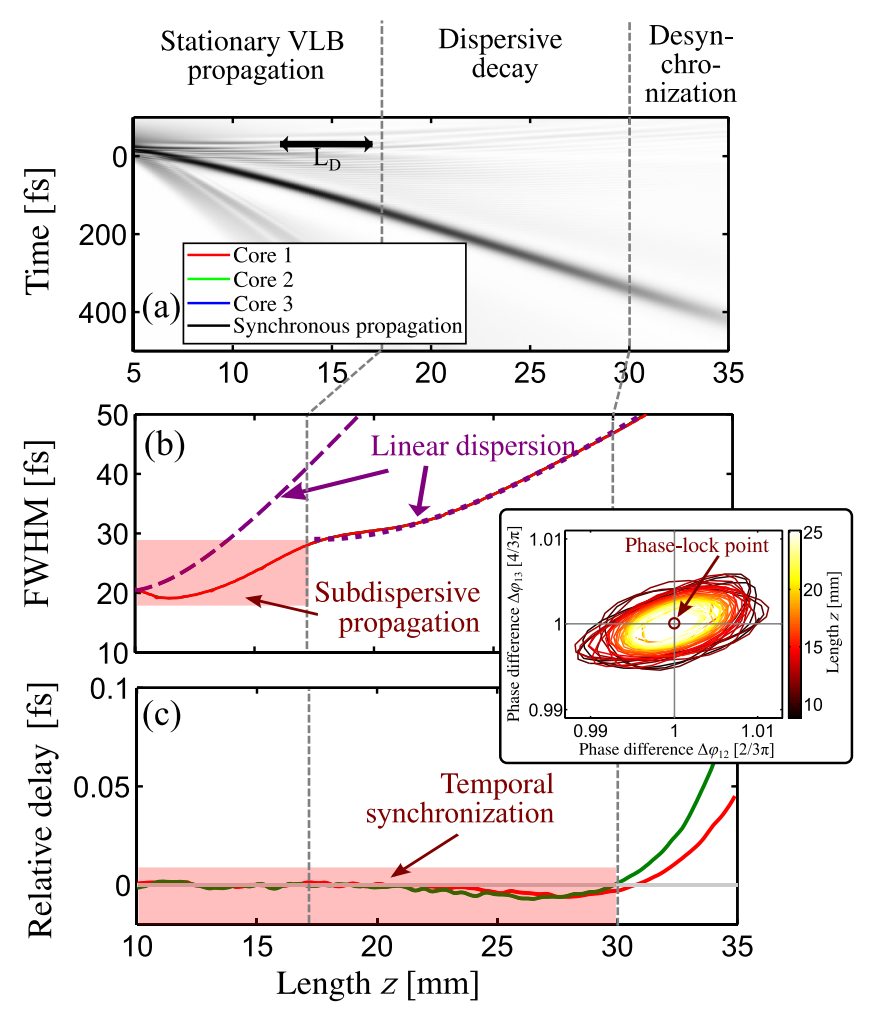

FIG. 5. Simulation of VLB propagation for excitation with three identical 50 -fs pulses with $2 \pi / 3$ relative phase shift and total energy $E=265 \mathrm{~nJ}$. (a) Pulse power as a function of time and sample length in the three central cores of the array. Each core's instantaneous optical power is coded into the image's RGB channel-gray denotes synchronous propagation. (b) FWHM in the central cores. The pulse, represented by a dashed line, shows hypothetical linear dispersion. The dotted purple line shows that linear dispersive broadening describes the pulse behavior well after $Z>17 \mathrm{~mm}$. (c) Peak delay of the pulse in the second and third cores with respect to the first. The desynchronization onset is visible by growth of temporal synchronization for large distances. Inset: Phase shift of the pulse in the first core with respect to the second and third. The phase-lock point (red circle) is an attractor, approached by the system in a damped orbit. (The propagation length is color coded.)

cores are also phase locked to a nonlinear attractor at relative phase shifts of $2 \pi / 3$ and $4 \pi / 3$, respectively [see the inset of Fig. 5]. Initial pulse contraction takes roughly $Z_{\min }=5 \mathrm{~mm}$, such that we observe VLB propagation for $L_{\mathrm{VLB}}=12 \mathrm{~mm}$, equating into $L_{\mathrm{VLB}}=2.5 L_{D}$, where $L_{D}$ is the dispersion length. Although $L_{\mathrm{VLB}}$ is just a bit more than one-half of the array's diffraction length $L_{\text {diff }}=\pi /\left(2 \times 6^{1 / 2} c\right)=23 \mathrm{~mm}$, one needs to keep in mind that the appropriate spatial scale $[31,38]$ for LBs is given by $\pi /(20 c) \approx L_{D} \ll L_{\text {diff }}$, and therefore VLBs are also spatially robust.

Note that all parameters are only given after $Z=$ $10 \mathrm{~mm}$, as parameter extraction is unreliable for the range of $5 \mathrm{~mm}<Z<10 \mathrm{~mm}$, because the VLB is not yet separated from the dispersive background. More details of the 
temporal dynamics in the three main cores are shown in Fig. 5(a).

For propagation lengths larger than $Z_{\max }$, we observe the same Raman-induced, redshift-mediated decay [31] that we previously reported for single LBs accompanied by linear pulse broadening [Fig. 5(c)]. There, we found stationary propagation for twice the distance. We attribute this difference to the higher powers required for stable VLBs, leading to an increased redshift rate and reduced lifetime [38].

To analyze the impact of inevitable excitation asymmetries, we reexamine the range of critical energies, with a 5\% energy reduction in the second core and a $10 \%$ reduction in the third core with respect to the first. Linear excitation with $E \ll 100 \mathrm{~nJ}$ does not produce VLBs, but no temporal desynchronization or other symmetry breaking is observed due to the absence of nonlinearity. If the input energy is too large $E>300 \mathrm{~nJ}$, we observe rapid temporal and energetic desynchronization and the generation of individual LBs.

If the energy is, however, in the range of $260 \mathrm{~nJ}<E<$ $300 \mathrm{~nJ}$ [Fig. 6(a)], we observe temporal synchronization to less than 5 fs for $Z<15 \mathrm{~mm}$ [Figs. 6(b) and 6(e)] with phase synchronization to less than $10 \%$ of the respective phase difference of $2 \pi / 3$ [see Fig. 6(f)]. As opposed to higher powers, the peaks exchange energy periodically [Figs. 6(c) and 6(d)] and approach equilibrium for $Z<15 \mathrm{~mm}$. At longer lengths, desynchronization into multiple individual LBs is observed.

Thus, for a limited range of energies, VLB propagation is possible in samples shorter than $15 \mathrm{~mm}$, even with slightly asymmetric excitation and a realistic propagation model. Longer samples and larger energies do not support VLBs but desynchronized individual LBs.

Experimental characterization is carried out with the imaging cross-correlation method established previously [31,36-39]. Details of the method are described in Appendix C. An overview of the setup is given in Fig. 1. The cross correlator is augmented by a self-referencing pulse shaper [48], allowing us to improve the temporal resolution of the cross correlator to 20 fs with approximately 10 -fs jitter. Pulse compression comes at the expense of reduced beam quality, leading to spatial variation in
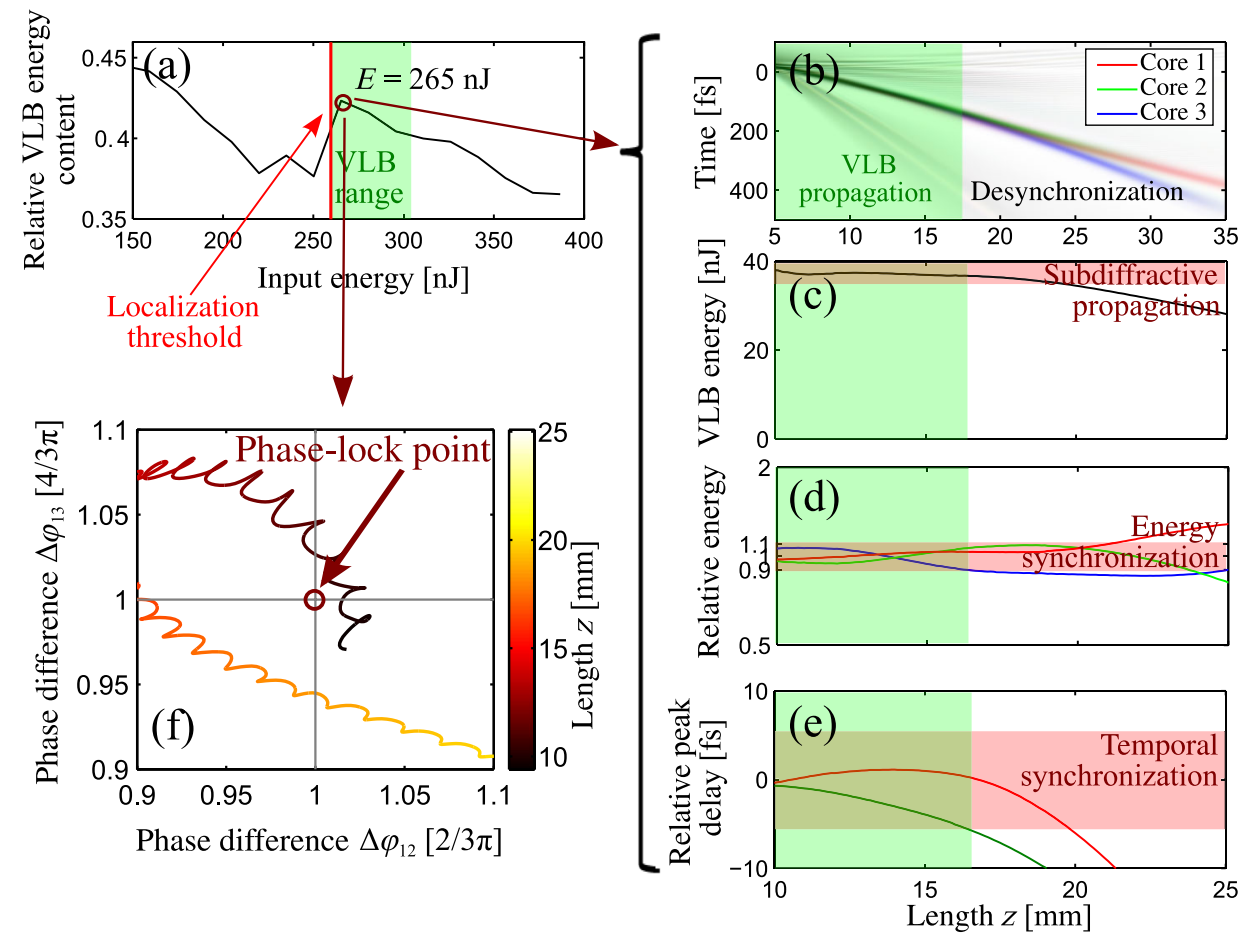

FIG. 6. Simulation of VLB propagation for excitation with three 50 -fs pulses with $2 \pi / 3$ relative phase shift and initial peak power differences of $10 \%$ in the second and 5\% in the third cores. (a) Relative energy content in the main pulse of the three central cores vs input energy. (b)-(f) Detailed results for an excitation energy $E=265 \mathrm{~nJ}$. (b) Instantaneous power as a function of time and sample length in the three central cores. Each core's power is coded into one of the image's RGB channels. Gray denotes synchronous propagation. Regions of stationary VLB propagation are represented in green. Regions with desynchronization are represented in white. (c) VLB energy content during propagation. The subdiffractive region $(Z<20 \mathrm{~mm})$ is represented in red. (d) Relative energy content of each of the cores with respect to the average energy content. Regions with energy synchronization $(Z<17 \mathrm{~mm})$ are represented in red. (e) Peak delay of the pulse in the second and third cores with respect to the first. Regions with temporal synchronization $(Z<17 \mathrm{~mm})$ are marked in red. (f) Phase shift of the first core with respect to the second and third cores during propagation. The phase-lock point (red circle) is an attractor, approached by the state of the system in a damped orbit as long as $Z<17 \mathrm{~mm}$. (The propagation length is color coded.) 


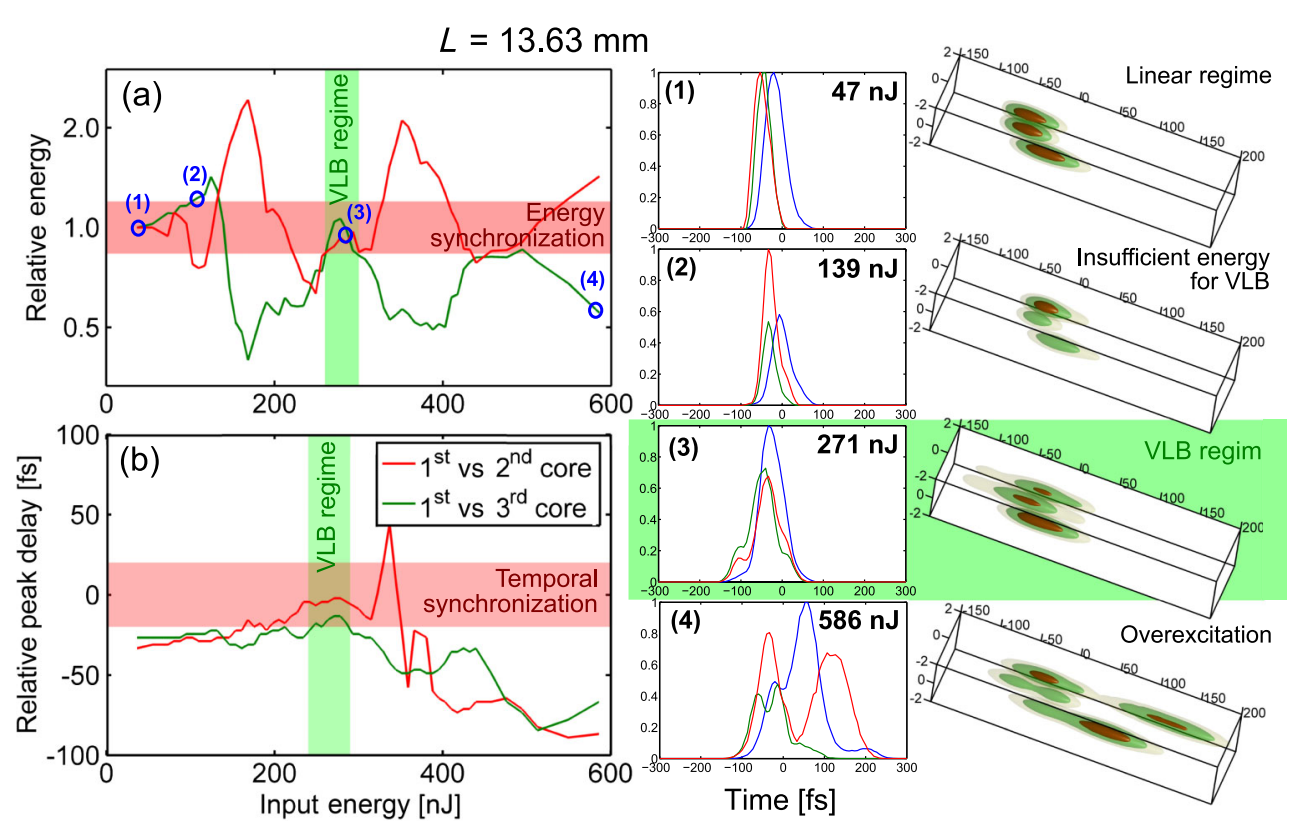

FIG. 7. Experimental data for a short sample. (a) Relative energy content of the second and third cores with respect to the first vs excitation energy. (b) Relative peak delay of the second and third cores with respect to the first vs excitation energy. Red bands represent regions of energetic and temporal synchronization defined by the respective measurement accuracies. Green bands represent regions of VLB existence as judged by predicted energy ranges and by temporal and energetic synchronization. (1)-(4), left column: Spatiotemporal cross-correlation traces at the location of the three central core modes at energies denoted in (a). (1)-(4), right column: 3D cross-correlation, isointensity plots of the same data. The green box denotes a typical VLB correlation.

the sum-frequency generation efficiency and a power uncertainty of up to $10 \%$.

Measurements are taken for samples of 13.63-, 16.88-, and 29.92-mm length, chosen such that the short sample facilitates the measurement of VLBs, whereas the long samples allow the characterization of decay into multiple desynchronized LBs.

Results for the short sample are depicted in Fig. 7. Figure 7(a) displays the relative energy content of the second and third cores with respect to the first. Similarly, Fig. 7(b) displays the relative delay of the peaks of the second and third pulses with respect to the first. Energetic and temporal synchronization and thus VLB observation are achieved if both curves enter the red bands, whose heights are determined by the respective measurement uncertainties. Synchronization is observed for an energy range of $260 \mathrm{~nJ}<E<300 \mathrm{~nJ}$, in agreement with the prediction.

Lower input energies produce nonsymmetric sets of pulses, with a pronounced single LB in one of the channels, as predicted. At very low energies, no nonlinear reshaping is observed and all pulses are of more or less equal power and almost synchronized due to the absence of nonlinear reshaping. At higher input energies, the pulses are again desynchronized. They form a set of multiple, temporally independent, single LBs, as predicted. The right part of Fig. 7 shows cross-correlation traces in 2D and 3D representations at input energies marked with circles in Fig. 7(a). The energies are chosen to represent all four of the discussed scenarios.
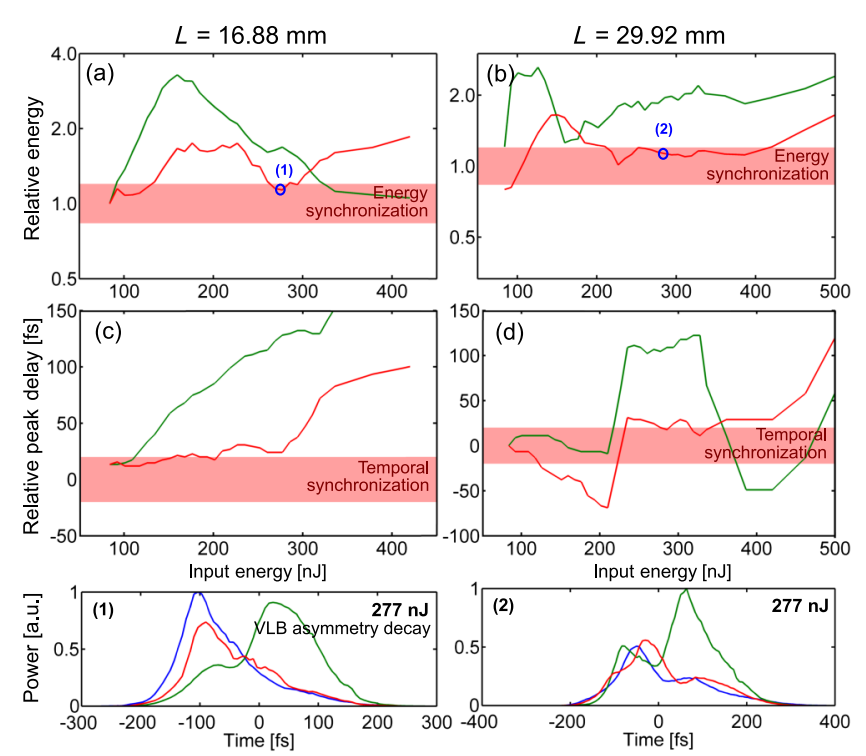

FIG. 8. Experimental data for longer samples. Left: A sample with $L=16.88 \mathrm{~mm}$, just longer than the maximal VLB propagation range. Right: A sample with $L=29.92 \mathrm{~mm}$. (a),(b) Relative energy content of the second and third cores with respect to the first vs excitation energy. (c),(d) Relative peak delay of the second and third cores with respect to the first vs excitation energy. The red bands represent regions of energetic and temporal synchronization defined by the respective measurement accuracies. (1),(2) Spatiotemporal cross-correlation traces at the location of the three central core modes at energies where VLB propagation is measured for shorter samples. 
Results for longer samples are depicted in Fig. 8. No synchronization takes place, and thus no VLBs are observed. It is interesting to note that the $16.88-\mathrm{mm}$ sample, which is just a little bit longer than the predicted maximum VLB range of $Z=15 \mathrm{~mm}$, exhibits an onset of synchronization, with almost identical energies [Fig. 8(a)] but with nonzero time delay [Fig. 8(b)], which is in accordance with simulations, predicting decay into temporally desynchronized LBs at maximum VLB propagation length. For much longer samples [Figs. 8(b) and 8(c)], even more asymmetry is observed and an onset of synchronization is no longer visible. Subfigures (1) and (2) of Fig. 8 depict exemplary cross-correlation data in the VLB energy range.

\section{CONCLUSIONS}

We have observed discrete VLBs, the arguably most complex, nonlinear, solitary, spatiotemporal wave packets reported so far. VLBs are a bound state of three temporally synchronized pulses with a respective phase shift of $2 \pi / 3$ in a triangular configuration. VLBs are solitary waves, stabilized by orbital angular momentum. They propagate in a discrete environment that interacts nontrivially with the vorticity of the light, even in the linear regime.

As opposed to ordinary LBs, VLBs are of semistable nature and decay into a set of desynchronized LBs after considerable propagation length. Experimental data and realistic numerical modeling show that VLBs are robust against higher-order effects and asymmetric excitation, with a roughly twofold decrease in stationary propagation length compared to LBs. They occur in a limited energy window, characterized by temporal and energetic synchronization up to a certain sample length. All experimental findings are in perfect agreement with numerical predictions and give insight into the overwhelmingly complex behavior and internal dynamics of VLBs.

These findings shed new light on effects to be discovered in the physics of high-dimensional, nonlinear excitations and will pave the way for a better understanding of nonlinear wave phenomena in many physical environments.

\section{APPENDIX A: ARRAY FABRICATION AND PROPERTIES}

The fiber arrays are constructed by a stack-and-draw process similar to photonic crystal fibers, with a solid core of increased refractive index instead of air holes [34,35]. The design is optimized for high regularity and negligible disorder $[49,50]$ over all of the 91 cores and lengths up to a few decimeters. The fiber used has a core radius of $r=10.3 \mu \mathrm{m}$ with a center-to-center distance of $\Lambda=34.7 \mu \mathrm{m}$. It is depicted in Fig. 9. The core is from pure silica, whereas the cladding is fluorine doped, yielding a refractive-index depression of $\Delta n=1.1 \times 10^{-3}$. The outer diameter is $670 \mu \mathrm{m}$ and can be varied in the drawing process with all other geometric parameters.

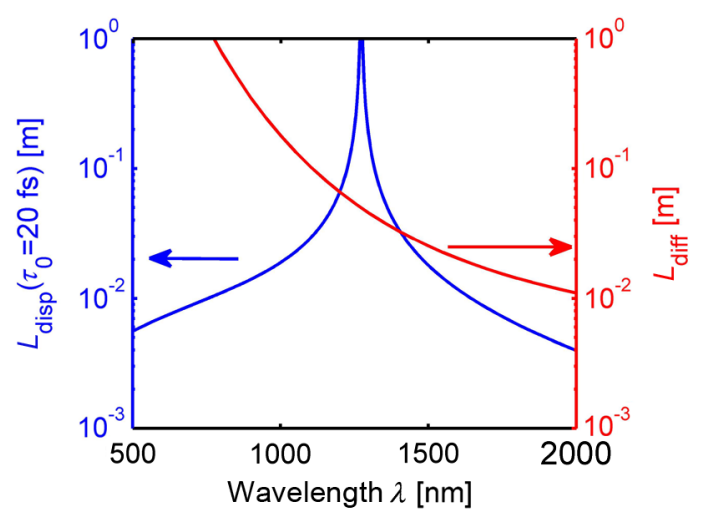

FIG. 9. Linear parameters of the fiber array as a function of wavelength. The blue line represents the dispersion length $L_{\text {disp }}=\tau_{0}^{2} /\left|\beta_{2}\right|^{2}$ with $\tau_{0}=20 \mathrm{fs}$, and the red line represents the diffraction length $L_{\text {diff }}=\pi /(2 \sqrt{6} c)$.

For the excitation wavelength of $\lambda=1550 \mathrm{~nm}$, this design is characterized by a waveguide coupling coefficient $c=$ $28.6 \mathrm{~m}^{-1}$, a dispersion of roughly $\beta_{2}=27 \times 10^{3} \mathrm{fs}^{2} / \mathrm{m}$, and a nonlinear coefficient of $\gamma=0.28 \times 10^{-3}(\mathrm{Wm})^{-1}$. Whereas the former two quantities $c$ and $\beta_{2}$ grow with increasing wavelength, $\gamma$ is decreasing, with the most drastic change [39] in the coupling constant $c$, as seen in Fig. 9.

\section{APPENDIX B: EXCITATION OF LBs}

To excite VLBs, we generate an input field with the desired angular momentum and a modulus that guarantees sufficient overlap with the modal fields in the three main waveguides. Such an input field is achieved by the placement of a threefold phase plate [44] before the focusing lens. The phase plate is made of silica and consists of three $120^{\circ}$ arcs fabricated in an etching process. It is depicted in Fig. 4(a). The thickness increases by $2 / 3 \lambda /(n-1)$ per arc. $n$ is the refractive index of silica, and $\lambda=1550 \mathrm{~nm}$ is the center wavelength of the excitation, which is chosen in the anomalous-dispersion regime of the array far away from the zero-dispersion wavelength, facilitating soliton propagation and impeding supercontinuum generation.

The focal pattern of the phase plate inherits the angular momentum and discrete nature of the phase plate. It is depicted in Figs. 4(a) and 4(b) and consists of three slightly distorted foci with $2 / 3 \pi$ relative phase shift. Their diameters and half-distances are approximately the same as the diameter of the focal spot without the phase plate. Both quantities can be manipulated by selection of an appropriate focusing lens. The similarity of the focal diameter and focal half-distances fits very well to the geometry of the fiber array, having a modal diameter of roughly $16 \mu \mathrm{m}$ and an array pitch of $\Lambda=34.9 \mu \mathrm{m}$. An excitation efficiency of roughly $65 \%$ is achieved. To properly orient the array with respect to the focal pattern, we place the array in a 
rotatable fiber holder. Moreover, we have used simulations to check that the pulse has no considerable spatiotemporal distortion.

\section{APPENDIX C: ANALYSIS OF LBs-THE IMAGING CROSS CORRELATOR}

The spatiotemporal light distribution $A_{\text {sig }}(x, y, t)$, which is leaving the waveguide array, passes an output lens, where it is magnified and imaged onto a thin slice of a beta-barium-borate (BBO) crystal. The crystal is cut and oriented for the sum-frequency generation of signal photons at $\lambda_{\text {sig }}=1550 \mathrm{~nm}$ and reference photons at $\lambda_{\text {ref }}=$ $800 \mathrm{~nm}$, generating photons at wavelengths of roughly $\lambda_{\mathrm{SF}}=527 \mathrm{~nm}$. The crystal width guarantees phase matching over a range of more than $500 \mathrm{~nm}$ and an angular acceptance range that is compatible with the possible range of incidence angles.

The reference pulse is supplied by a part of the pump pulse bypassing the experiment, propagating collinearly through the BBO together with the signal. See Fig. 1 for an overview of the experiment. The pulse has a Gaussian shape with a duration of roughly $20 \mathrm{fs}$, formed by an adaptive, spectral pulse-shaping stage [48] not depicted in Fig. 1. Its diameter is larger than the signal image on the BBO. The relative delay $\tau$ of the reference pulse to the signal is tuned with a delay line. The motion of the delay line is synchronized with a CCD camera that records the sum-frequency signal with a second imaging lens. The sum-frequency signal $I_{\mathrm{SF}}(x, y, \tau)$ is therefore a threedimensional representation of the signal intensity $\left|A_{\text {sig }}(x, y, t)\right|^{2}$, convolved with the reference pulse shape. This scheme allows us to measure the spatiotemporal intensity distribution of the VLB with a resolution of 20 fs [36,37] and roughly 10 -fs timing jitter in parallel over the complete waveguide array. The measurement duration is only a few seconds. To avoid recording nonsum-frequency background, undesired wavelength components are blocked before the CCD.

\section{APPENDIX D: DEFINITION OF THE PERTURBATION}

Given is an initial state $\tilde{a}_{n m}(t)$, which is a stationary solution to Eq. (1). We observe the evolution of the solution $a_{n m}(z, t)$ of the nonlinear Schrödinger equation Eq. (1), with $\tilde{a}_{n m}(t)$ as the initial state. According to the ansatz, the initial state $\left|a_{n m}(z, t)\right|$ is invariant during propagation, if the solution is stable; however, perturbation growth is observed for unstable solutions. We therefore define the quantity

$$
\log (\varepsilon)=\log \frac{\sum_{n m} \int_{-\infty}^{\infty}\left|a_{n m}(z, t)\right|-\left|\tilde{a}_{n m}(t)\right| d t}{\sum_{n m} \int_{-\infty}^{\infty}\left|\tilde{a}_{n m}(t)\right| d t}
$$

to measure how the initial state is perturbed by the propagation.
[1] M. A. Ablowitz and P. A. Clarkson, Solitons, Nonlinear Evolution Equations and Inverse Scattering (Cambridge University Press, Cambridge, England, 1991).

[2] Y. Kivshar and G. Agrawal, Optical Solitons (Academic, San Diego, CA, 2003).

[3] E. A. Ostrovskaya and Yu. S. Kivshar, Matter-Wave Gap Solitons in Atomic Band-Gap Structures, Phys. Rev. Lett. 90, 160407 (2003).

[4] T. Dauxois and M. Peyard, Physics of Solitons (Cambridge University Press, Cambridge, England, 2006).

[5] A. L. Hodgkin and A.F. Huxley, A Quantitative Description of Membrane Current and Its Application to Conduction and Excitation in Nerve, J. Physiol. 117, 500 (1952).

[6] R. Clarke, R. Smith, and D. Reid, The Morning Glory of the Gulf of Carpentaria: An Atmospheric Undular Bore, Mon. Weather Rev. 109, 1726 (1981).

[7] A. Hasegawa and F. Tappert, Transmission of Stationary Nonlinear Optical Pulses in Dispersive Dielectric Fibers. I. Anomalous Dispersion, Appl. Phys. Lett. 23, 142 (1973).

[8] G. Agrawal, Nonlinear Fiber Optics (Academic, Boston, 2001).

[9] D. N. Christodoulides, F. Lederer, and Y. Silberberg, Discretizing Light Behaviour in Linear and Nonlinear Waveguide Lattices, Nature (London) 424, 817 (2003).

[10] Y. V. Kartashov, B. A. Malomed, and L. Torner, Solitons in Nonlinear Lattices, Rev. Mod. Phys. 83, 247 (2011).

[11] F. Lederer, G. I. Stegeman, D. N. Christodoulides, G. Assanto, M. Segev, and Y. Silberberg, Discrete Solitons in Optics, Phys. Rep. 463, 1 (2008).

[12] D. N. Christodoulides and R.I. Joseph, Discrete SelfFocusing in Nonlinear Arrays of Coupled Waveguides, Opt. Lett. 13, 794 (1988).

[13] H. S. Eisenberg, Y. Silberberg, R. Morandotti, A. R. Boyd, and J.S. Aitchison, Discrete Spatial Optical Solitons in Waveguide Arrays, Phys. Rev. Lett. 81, 3383 (1998).

[14] B. A. Malomed, D. Mihalache, F. Wise, and L. Torner, Spatiotemporal Optical Solitons, J. Opt. B 7, R53 (2005).

[15] F. Eilenberger, A. Szameit, and T. Pertsch, Transition from Discrete to Continuous Townes Solitons in Periodic Media, Phys. Rev. A 82, 043802 (2010).

[16] A. Szameit, J. Burghoff, T. Pertsch, S. Nolte, A. Tünnermann, and F. Lederer, Two-Dimensional Soliton in Cubic $f_{s}$ Laser Written Waveguide Arrays in Fused Silica, Opt. Express 14, 6055 (2006).

[17] B. A. Malomed and P.G. Kevrekidis, Discrete Vortex Solitons, Phys. Rev. E 64, 026601 (2001).

[18] D. Neshev, E. Ostrovskaya, Yu. Kivshar, and W. Krolikowski, Spatial Solitons in Optically Induced Gratings, Opt. Lett. 28, 710 (2003).

[19] J. W. Fleischer, M. Segev, N. K. Efremidis, and D. N. Christodoulides, Observation of Two-Dimensional Discrete Solitons in Optically Induced Nonlinear Photonic Lattices, Nature (London) 422, 147 (2003).

[20] J. W. Fleischer, T. Carmon, M. Segev, N. K. Efremidis, and D. N. Christodoulides, Observation of Discrete Solitons in Optically Induced Real Time Waveguide Arrays, Phys. Rev. Lett. 90, 023902 (2003). 
[21] D. N. Neshev, T. J. Alexander, E. A. Ostrovskaya, and Yu. S. Kivshar, Observation of Discrete Vortex Solitons in Optically Induced Photonic Lattices, Phys. Rev. Lett. 92, 123903 (2004).

[22] B. Terhalle et al., Observation of Double-Charge Discrete Vortex Solitons in Hexagonal Photonic Lattices, Phys. Rev. A 79, 043821 (2009).

[23] D. Cheskis, S. Bar-Ad, R. Morandotti, J. S. Aitchison, H. S. Eisenberg, Y. Silberberg, and D. Ross, Strong Spatiotemporal Localization in a Silica Nonlinear Waveguide Array, Phys. Rev. Lett. 91, 223901 (2003).

[24] A. V. Gorbach et al., Spatiotemporal Nonlinear Optics in Arrays of Subwavelength Waveguides, Phys. Rev. A 82, 041802(R) (2010).

[25] P. Di Trapani, W. Chinaglia, S. Minardi, A. Piskarskas, and G. Valiulis, Observation of Quadratic Optical Vortex Solitons, Phys. Rev. Lett. 84, 3843 (2000).

[26] L. Allen, M. W. Beijersbergen, R. J. C. Spreeuw, and J. P. Woerdman, Orbital Angular Momentum of Light and the Transformation of Laguerre-Gaussian Laser Modes, Phys. Rev. A 45, 8185 (1992).

[27] J. Wang et al., Terabit Free-Space Data Transmission Employing Orbital Angular Momentum Multiplexing, Nat. Photonics 6, 488 (2012).

[28] Y. Silberberg, Collapse of Optical Pulses, Opt. Lett. 15, 1282 (1990).

[29] D. Mihalache, Linear and Nonlinear Light Bullets: Recent Theoretical and Experimental Studies, Romanian J. Phys. 57, 352 (2012).

[30] A. B. Aceves and C. De Angelis, Spatiotemporal Pulse Dynamics in a Periodic Nonlinear Waveguide, Opt. Lett. 18, 110 (1993).

[31] S. Minardi et al., Three-Dimensional Light Bullets in Arrays of Waveguides, Phys. Rev. Lett. 105, 263901 (2010).

[32] C. J. Benton, A. V. Gorbach, and D. V. Skryabin, Spatiotemporal Quasisolitons and Resonant Radiation in Arrays of Silicon-on-Insulator Photonic Wires, Phys. Rev. A 78, 033818 (2008).

[33] C.J. Benton and D. V. Skryabin, Coupling Induced Anomalous Group Velocity Dispersion in Nonlinear Arrays of Silicon Photonic Wires, Opt. Express 17, 5879 (2009).

[34] U. Röpke, H. Bartelt, S. Unger, K. Schuster, and J. Kobelke, Two-Dimensional High-Precision Fiber Waveguide Arrays for Coherent Light Propagation, Opt. Express 15, 6894 (2007).

[35] U. Röpke, H. Bartelt, S. Unger, K. Schuster, and J. Kobelke, Fiber Waveguide Arrays as Model System for Discrete Optics, Appl. Phys. B 104, 481 (2011).

[36] M. A. C. Potenza, S. Minardi, J. Trull, G. Blasi, D. Salerno, A. Varanavičius, A. Piskarskas, and P. Di Trapani, Three
Dimensional Imaging of Short Pulses, Opt. Commun. 229, 381 (2004).

[37] S. Minardi, J. Trull, and M. A. C. Potenza, Holographic Properties of Parametric Image Conversion for Spatiotemporal Imaging of Ultrashort Laser Pulses, J. Holography Speckle 5, 85 (2009).

[38] F. Eilenberger et al., Evolution Dynamics of DiscreteContinuous Light Bullets, Phys. Rev. A 84, 013836 (2011).

[39] F. Eilenberger, S. Minardi, A. Szameit, U. Röpke, J. Kobelke, K. Schuster, H. Bartelt, S. Nolte, A. Tünnermann, and T. Pertsch, Light Bullets in Waveguide Arrays: SpacetimeCoupling, Spectral Symmetry Breaking and Superluminal Decay, Opt. Express 19, 23171 (2011).

[40] L. Bergé, Wave Collapse in Physics: Principles and Applications to Light and Plasma Waves, Phys. Rep. 303, 259 (1998).

[41] F. Eilenberger, M. Bache, S. Minardi, and T. Pertsch, Temporal Switching Induced by Cascaded Third Order Nonlinearity, Opt. Lett. 37, 5109 (2012).

[42] H. Leblond, B. A. Malomed, and D. Mihalache, Spatiotemporal Vortices in Optical Fiber Bundles, Phys. Rev. A 77, 063804 (2008).

[43] H. Leblond, B. A. Malomed, and D. Mihalache, Spatiotemporal Vortex Solitons in Hexagonal Arrays of Waveguides, Phys. Rev. A 83, 063825 (2011).

[44] K. Sueda, G. Miyaji, N. Miyanaga, and M. Nakatsuka, Laguerre-Gaussian Beam Generated with a Multilevel Spiral Phase Plate for High Intensity Laser Pulses, Opt. Express 12, 3548 (2004).

[45] S. Minardi and T. Pertsch, Interferometric Beam Combination with Discrete Optics, Opt. Lett. 35, 3009 (2010).

[46] M. Kolesik and J. V. Moloney, Nonlinear Optical Pulse Propagation Simulation: From Maxwell's to Unidirectional Equations, Phys. Rev. E 70, 036604 (2004).

[47] I. Babushkin, A. Husakou, J. Herrmann, and Yu. S. Kivshar, Frequency-Selective Self-Trapping and Supercontinuum Generation in Arrays of Coupled Nonlinear Waveguides, Opt. Express 15, 11978 (2007).

[48] V. V. Lozovoy, I. Pastirk, and M. Dantus, Multiphoton Intrapulse Interference. IV. Ultrashort Laser Pulse Spectral Phase Characterization and Compensation, Opt. Lett. 29, 775 (2004).

[49] T. Pertsch, U. Peschel, J. Kobelke, K. Schuster, H. Bartelt, S. Nolte, A. Tünnermann, and F. Lederer, Nonlinearity and Disorder in Fiber Arrays, Phys. Rev. Lett. 93, 053901 (2004).

[50] T. Schwartz, G. Bartal, S. Fishman, and M. Segev, Transport and Anderson Localization in Disordered Two-Dimensional Photonic Lattices, Nature (London) 446, 52 (2007). 